\title{
The Fly's Eye Camera System - an instrument design for large étendue time-domain survey
}

\author{
András Pál ${ }^{1,2, \star}$, László Mészáros ${ }^{1,2}$, Gergely Csépány ${ }^{1}$, Attila Jaskó ${ }^{1}$, Ferenc Schlaffer ${ }^{3}$, Krisztián \\ Vida $^{1}$, György Mezô ${ }^{1}$, László Döbrentei ${ }^{1}$, Ernő Farkas ${ }^{1}$, Csaba Kiss ${ }^{1}$, Katalin Oláh ${ }^{1}$, and Zsolt Regály ${ }^{1}$ \\ 1 MTA Research Centre for Astronomy and Earth Sciences, Konkoly Thege Miklós út 15-17, Budapest, H-1121, Hungary \\ 2 Department of Astronomy, Loránd Eötvös University, Pázmány Péter sétány 1/A, Budapest H-1117, Hungary \\ 3 MTA Research Centre for Astronomy and Earth Sciences, Csatkai E. u. 6-8, Sopron, H-9400, Hungary
}

Received 15 Nov 2012, accepted 15 Nov 2012

Published online later

Key words Editorial notes - instruction for authors

In this paper we briefly summarize the design concepts of the Fly's Eye Camera System, a proposed high resolution all-sky monitoring device which intends to perform high cadence time domain astronomy in multiple optical passbands while still accomplish a high étendue. Fundings have already been accepted by the Hungarian Academy of Sciences in order to design and build a Fly's Eye device unit. Beyond the technical details and the actual scientific goals, this paper also discusses the possibilities and yields of a network operation involving $\sim 10$ sites distributed geographically in a nearly homogeneous manner. Currently, we expect to finalize the mount assembly - that performs the sidereal tracking during the exposures - until the end of 2012 and to have a working prototype with a reduced number of individual cameras sometimes in the spring or summer of 2013.

(c) 2012 WILEY-VCH Verlag GmbH \& Co. KGaA, Weinheim

\section{Introduction}

Astrophysical phenomena take place on a wide range of timescales. From the shortest millisecond signals of pulsars up to the lifetime of stars, that can be comparable to the age of the Universe, there is an astonishing span of $\sim 20$ magnitudes. The key to unveil the physical processes beyond these phenomena is to monitor the alterations of observable quantities, such as flux. Although some of the processes have their own characteristic timescales, most of the complex systems exhibit variations on a broader temporal spectrum. These complex systems show signs of periodic, quasi-periodic and sudden transient, eruptive processes. The observed timescales imply not only the possible durations of matter rearrangement whatever is the reason behind, but constrain the physical backgrounds of the variabilities of the observed systems. Hence, persistent monitoring of such "astrophysical laboratories" helps us to understand how stars evolve, and from a wider perspective, how planetary systems and even our Solar System develop from their early stages of life until its end.

Astronomical surveys require a complex optical and detector system to cover a large field-of-view (FOV), which often pairs with large light collecting area. The cumulative light collecting power, known as the étendue defines how effective a certain instrument is for survey purposes. By following the astronomical scientific discovery orientations, as one of these is the time-domain astronomy (see Blandford et

Corresponding author: e-mail: apal@ flyseye.net al. 2010), recent initiatives for survey projects highly focus on the most extensive ways of implementing instrumentation with high optical acceptance.

The aim of our plan is to develop and build an instrument coined as Fly's Eye Camera System that allows the continuous monitoring of optical sky variability. The timescale window in which the instrument will operate covers $\sim 6$ order of magnitudes: from the data acquisition cadence in the range of minutes up to the expected range of several years of operation.

The proposed design yields an étendue that is comparable to the currently operating survey programs, such as the highly successful Kepler space telescope (Borucki et al. 2007) and the ambitious Pan-STARRS project (Kaiser et al. 2002). The Fly's Eye Camera System is a "high cadence + low imaging resolution + large solid angle coverage" instrument. Unlikely to e.g. Kepler that uses a "high cadence + high imaging resolution + small solid angle coverage" setup and Pan-STARRS that provides a "small cadence + high imaging resolution + large solid angle coverage" combination, the Fly's Eye allows the monitoring of a presently unexplored range of the domain of astronomical events.

Persistent monitoring of several thousand bright, scientifically relevant systems can only be implemented by the means of smaller multiplexed instruments exploiting smaller imaging resolutions. In addition, transient detection in various known or undiscovered systems and statistical analysis is also feasible in this domain. Thus, such an instrument will provide a backbone of high resolution photometric, polarimetric, interferometric, infrared, spec- 
troscopic and space-borne follow-up measurements as well. An extended, nearly uniform geographical distribution of $8-10$ Fly's Eye units would result in a light-grasp power comparable to the Large Synoptic Survey Telescope (LSST, Ivezić et al. 2008). The LSST is the highest ranked ground based facility in the strategic roadmap of American astronomy for the next decade (Blandford et al. 2010). Furthermore, as it is discussed in more details later on, the Fly's Eye design will provide a continuous transition to the brighter targets from the fainter ones aimed to be observed by LSST.

As we will explain here, the design is both simple and robust (Sec. 2) to build a geographically extended network of this camera system, providing a more dense phase coverage of the observed events and a wider perspective to the sky (Sec. 3). The proposed scientific applications cover disciplines from within the nearby Solar System (including even the atmosphere of the Earth) up to extragalactic investigations (Sec. 4).

\section{Instrument design}

The advance in consumer and computer electronics in recent years allows us to build this Fly's Eye device from commercially available and well-tested components with parameters that would not have been possible even a few years ago. Hence, by exploiting these hardware and optics, it is possible to design cost-effective instrumentation for scientific purposes. In the following, we detail the properties of specific cameras and lenses available in the marked from which the proposed design can easily be built and deployed.

The 19 cameras are mounted on a fixed assembly, i.e., the relative positions and field rotation angles are also kept fixed throughout the observations. We intend to employ the very recent and compact model ML-16803 of the FLI company and standard, commercially available Canon lenses with the focal length of $f=85 \mathrm{~mm}$ with a rather fast focal ratio of $f / 1.2$. This FOV will cover the sky all above the $h \geq 30^{\circ}$ horizontal altitude (i.e. half of the whole visible celestial sphere, up to airmasses $A \leq 2$ ), allowing the persistent survey of the sky with moderate imaging resolution. According to the specifications of the $4 \mathrm{k} \times 4 \mathrm{k} \mathrm{KAF}$ 16803 detectors that are used by the ML-16803 cameras, this setup yields a resolution of $22^{\prime \prime} /$ pixel. The resulting cumulative optical light collecting power (i.e. the étendue) of the system will be nearly $35-40 \mathrm{deg}^{2} \mathrm{~m}^{2}$, depending on the vignetting of the lenses. This large value places the whole device among the group of the instruments with the highest optical attendance. The expected photometric precision expecting a cadence of 3 minutes is $4-500 \mathrm{ppm}$ for point sources of $r=10$ magnitude while a precision of $10 \%$ is expected at $r=15$. The latter number can also be interpreted as a detection threshold with $\mathrm{S} / \mathrm{N}=10$. Due to its resolution on the faint end, the photometric uncertainties are because of the confusion of nearby sources. The astrometric and photometric analysis of imaging data is performed with the FITSH package ${ }^{1}$, found to be rather effective in widefield optical variability surveys (see Pál 2009, Pál 2012).

A visualization of the mount design concept is displayed in Fig. 1 along with the resulting sky coverage and the FOV of each camera-lens pair. The main concept of the camera design are a) to minimize the number of moving parts and b) not to use specialized, uniquely designed and/or manufactured mechanical, optical or electronic components in the device. This second "rule of thumb" allows us to have spare parts of all of the necessary components that can be replaced instantly upon a failure and hence, does not add a significant investment and maintenance cost. Therefore, one can expect a smooth and continuous operation of the camera system, since the simple design concepts allows a fast replacement of broken parts.

The camera platform will minimize the unique types of moving parts. In the history of automated telescope surveys we clearly identified this to be the main problem of reliable operation. The mount is planned to be based on a hexapod-design (also known as Steward-platform ${ }^{2}$, that requires only identical mechanical elements and allows the desired motion independently of the placement of the mount support base. See also the left panel of Fig. 1 of a schematic drawing of the hexapod mount.

Sidereal tracking of the camera platform is performed during the exposures while the local first equatorial coordinates of the platform would exactly be the same throughout the subsequent exposures. Therefore, during the image readout, the whole platform is slewed back to its initial position and performs the same apparent path in the next exposure and so on. The design for arranging the cameras on the platform resembles the PASS instrument (Deeg et al. 2004). In the PASS design, the cameras are fixed to the ground, hence there stellar images show trails: the essential improvement to PASS is the implementation of the sidereal tracking.

Since parameterization of the rotation via the pitch, roll and yaw axes does not imply any singularity (like a gimbal lock) for arbitrary small rotations (i.e. when the total rotation is $\rho \ll 90^{\circ}$ ), this mount can be employed for sidereal tracking on arbitrary geographical latitudes. Indeed, installing the mount to the poles of the Earth yields a pure yaw rotation while installing the mount on the equator yields a pure roll rotation (expecting the $x \pm$ axis pointing to northsouth). On "temperate" latitudes, the sidereal rotation will be a combination of yaw and roll, while the pitch rotation is required only for correcting the polar alignment. The if the tracking accuracy should be one tenth of the pixel resolution, $2^{\prime \prime} \approx 10 \mu \mathrm{rad}$, then the actuators must be controlled with a precision of $\approx 5 \mu \mathrm{m}$ (expecting a characteristic platform size and/or a retracted actuator length of $0.5 \mathrm{~m}$ ). This is feasible by our choice from the commercially available actuators.

\footnotetext{
1 http://fitsh.szofi.net/

${ }^{2}$ See e.g. http://en.wikipedia.org/wiki/Stewart_platform
} 

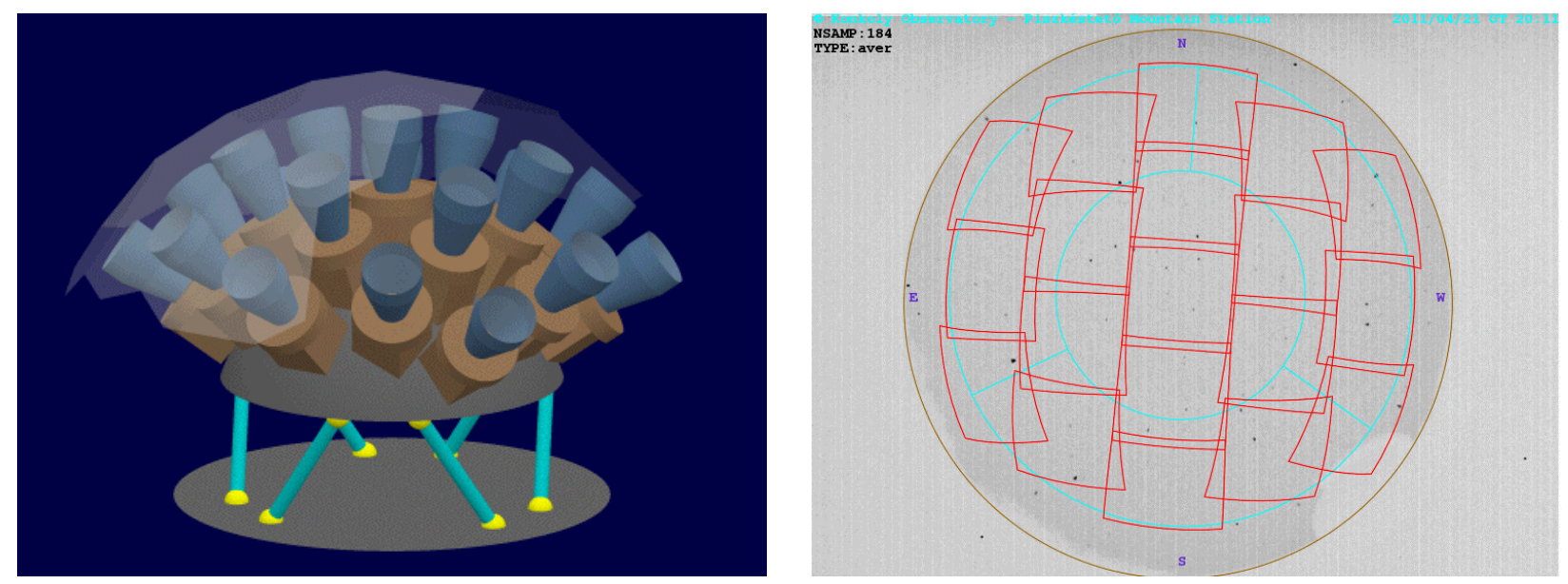

Fig. 1 Left panel: a simple visualization of the camera mount. The payload platform - on which the 19 FLI Microline cameras have been mounted - is shown to scale. Hence, the diameter of the platform is approximately $55 \mathrm{~cm}$ while the effective diameter including the cameras and lenses is nearly $1 \mathrm{~m}$. The lower, fixed platform and the hexapod strut drawings are merely figurative, but the expected distance between the two platforms are roughly $25-30 \mathrm{~cm}$ (as it is implied by the figure scale). The mosaic dome is partly shown also to scale, as a transparent set of hexagonal elements. The size of the hexagonal elements are roughly $23-25 \mathrm{~cm}$. Right panel: the FOV of the 19 cameras shown on an (inverted) all-sky image. Assuming a focal distance of $85 \mathrm{~mm}$ for the lenses and ML-16803 cameras as detectors, the FOV of each camera - lens pair will be roughly $26^{\circ}$. The placement and field orientation of the cameras are exactly the same as it is shown in the left panel. The two concentric circles mark the $30^{\circ}$ and $60^{\circ}$ horizontal altitudes.

\section{Further developments}

Since the Fly's Eye camera observes the sky above an altitude of 30 degrees, it simultaneously monitors almost exactly the one fourth of the whole celestial sphere. From a temperate geographical latitude (e.g. from Hungary, at $\varphi \approx 47^{\circ}$ ), the Sun is below the horizon more than $12^{\circ}$ within a time fraction of close to 0.4 on average throughout a year (but do not differ significantly on other geographical locations). Hence, the system observes approximately the $(1 / 4) \cdot 0.4=10 \%$ of the detectable events. A natural way of increase this ratio is to install similar devices at other locations on Earth. In addition, monitoring the sky simultaneously from distinct (and far) locations significantly decreases the one-day aliases in the phase domains of periodic events. Moreover, synchronization in image acquisition also aids the accurate data reduction since the overlapping regions observed by distinct devices have to be exactly the same. This approach makes the characterization of systematic noise sources much more easier. For instance, such a synchronization can be accomplished by starting the exposures at every three minutes in Greenwich sidereal time.

Hence, we initiate further collaborations and seek for other types of grants that will cover the costs of building a network of these devices all around the world. Negotiations have also been started with the staff of Teide Observatory, Tenerife, Canary Islands. An example configuration of 9 devices located on various places on Earth with wellknown infrastructure suitable for installing astronomical instrumentation is displayed in Fig. 2 .

\section{Scientific goals}

The main goals of the proposed Fly's Eye Network project cover several topics in astrophysics. In the following, without attempting to be comprehensive, we list some of these sub-fields of astrophysics.

\subsection{Solar System}

Even with its moderate resolution, the Fly's Eye device is capable to detect meteors and map these tracks with an effective resolution of $\sim 10 \mathrm{~m} /$ pixel. Hence, and due to the large light collecting power of the device, a more accurate distribution of Solar System dust can be derived. In addition, for the bright-end of the main-belt asteroid family members, an unbiased sample will be available for their rotation and shape properties (see also Durech, Sidorin \& Kaasalainen 2010). These data are essential to understand the aspect of Solar System dynamics and, more importantly, its evolution.

Moreover, nearby flybys of small bodies that are potentially hazardous to the Earth can be traced (see e.g. the cases of $2005 \mathrm{YU}_{55}$ and $2012 \mathrm{BX}_{34}$ ). Due to the continuous sampling, such information is also recoverable in an a posteriori manner, i.e. when deeper surveys discover such an object and dynamical calculations confirm a former approach in the FOV of one or more Fly's Eye device. 


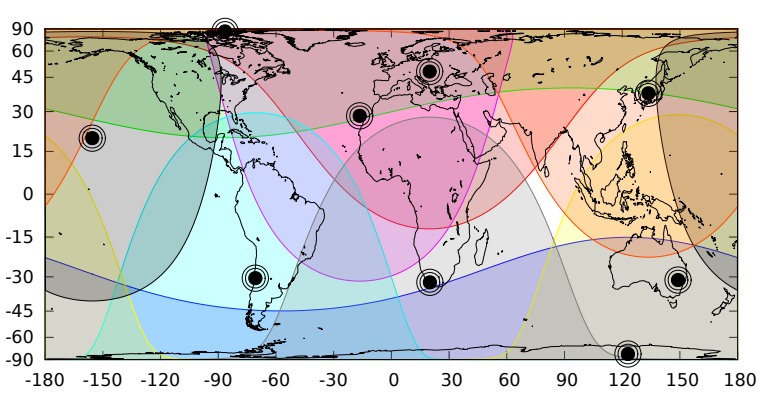

Fig. 2 An example configuration and the yielded visibility coverage of 9 Fly's Eye devices, distributed nearly homogeneously on the Earth (using Lambert cylindrical equal-area projection). See text for further details.

\subsection{Stellar and planetary systems}

Young stellar objects are complex astrophysical systems and show signs of both quasi-periodic and sudden transient, eruptive processes. By monitoring their intrinsic variability, one is able to obtain several constraints regarding to the ongoing processes (Hartmann \& Kenyon 1996. Herbig 2007. Ábrahám et al. 2009, Kóspál et al. 2011). Persistent monitoring of numerous young stellar objects or candidates for young stellar objects will reveal the nature of the currently unexplored domains of stellar birth. Since observing campaigns are organized mostly on daily or yearly basis, the behaviour of such systems is practically unknown on other timescales.

Stars with magnetic activity show photometric variability on all the time-domains of the planned instrument, from minutes through hours to years, just like the Sun does (Strassmeier 2009). Continuous monitoring of the sky opens up a new research area for active stars: the proposed device allows us to obtain good flare statistics since flares occur on minutes-hours timescale, and to monitor starspot evolution, differential rotation and activity cycles of the same star (see e.g. Hartmann et al. 2011, Walkowicz et al. 2011, Oláh et al. 2009, Vida et al. 2010).

Observations of eclipsing binaries provide direct measurements of stellar masses and radii that are essential to understand their evolution and even the basic physical processes ongoing in the stellar cores (Latham et al. 2009). Similarly to eclipsing binaries, transiting extrasolar planets are also expected to be discovered by the Fly's Eye Network, since instruments with nearly similar types of optics are found to be rather efficient (Pollacco et al. 2004, Bakos et al. 2004, Pepper et al. 2007, Pál et al. 2008, Pál 2009).

\subsection{In the extragalactic environment}

Continuous monitoring of brighter supernovae in nearby galaxies yield valuable data that can be exploited by combining other kind of measurements. The Fly's Eye camera is capable to observe the brightest supernovae directly even up to a month during their peak brightness (see e.g. Vinkó et al. 2012). By combining images, it is possible to go even deeper in brightness using more sophisticated ways of photometric techniques.

\section{Summary}

This paper briefly summarized both the instrument design concepts and the proposed series of scientific applications of an all-sky monitoring device named Fly's Eye Camera System. Due to the implementation of a hexapod-based camera platform, exactly the same instrument can be installed independently from the current geographical location as well as the whole setup does not need any kind of polar alignment. The robust design does not exploit any unique mechanical component hence the operations are highly fault tolerant and the maintenance is easy. The resulting étendue of the optical setup in a Fly's Eye unit is comparable to the largest available optical facilities, moreover, a global network of such devices yields an étendue that is similar to the current imaging system of the Large Synoptic Survey Telescope.

Acknowledgements. The "Fly's Eye" project is supported by the Hungarian Academy of Sciences via the "Lendület" grant LP2012$31 / 2012$. Additional support is also received via the ESA grant PECS 98073. We thank Hans Deeg (PI of the PASS project) for the useful discussions. We also thank the careful comments of the anonymous referee.

\section{References}

Ábrahám, P. et al.: 2009, Nature, 459, 224

Bakos, G. Á. et al.: 2004, PASP, 116, 266

Blandford, R. D. et al. (Committee for a Decadal Survey of Astronomy and Astrophysics): New Worlds, New Horizons in Astronomy and Astrophysics The National Academic Press, Washington $\mathrm{DC}, \mathrm{USA}^{3}$

Borucki, W. J. et al.: 2007, ASP Conf. Ser., 366, 309

Deeg, H. J.; Alonso, R.; Belmonte, J. A.; Alsubai, K.; Horne, K.; Doyle, L.: 2004 PASP, 116, 985

Ďurech, J.; Sidorin, V. \& Kaasalainen, M.: 2010, A\&A, 513, 46

Hartman, J. D. et al.: 2011, AJ, 141, 166

Hartmann, L. \& Kenyon, S. J.: 1996, ARA\&A, 34, 207

Herbig, G. H.: 2007, AJ, 133, 2679

Ivezić, Ž. et al: LSST: from Science Drivers to Reference Design and Anticipated Data Product $\$^{4}$

Kaiser, N. et al, 2002, in the proceedings of "Survey and Other Telescope Technologies and Discoveries“, edited by Tyson, J. Anthony; Wolff, Sidney. Proceedings of the SPIE, Volume 4836, pp. 154-164

Kóspál, Á. et al.: 2011, A\&A, 527, 133

Latham, D. W. et al.: 2009, ApJ, 704, 1107

Oláh, K. et al.: 2009, A\&A, 501, 703

Pál, A. et al.: 2008, ApJ, 680, 1450

Pál, A.: 2009, PhD thesis, Eötvös Loránd University, Budapest, Hungary (arXiv:0906.3486)

Pál, A.: 2012, MNRAS, 421, 1825

\footnotetext{
3 Available from http://www.nap.edu/catalog.php?record_id=12951

${ }^{4}$ Available from http://1sst.org/lsst/overview/
} 
Pepper, J. et al.: 2007, PASP, 119, 923

Pollacco, D. et al.: 2006, Ap\&SS, 304, 253

Strassmeier, K. G.: 2009, A\&ARv, 17, 251

Vida, K. et al.: 2010, AN, 331, 250

Vinkó, J. et al.: 2012, A\&A, accepted (arXiv:1111.0596)

Walkowicz, L. M. et al.: 2011, AJ, 141, 50 